\title{
Corpuscular Point of View to Explain Light's Properties
}

\author{
Jose L. Parra \\ Courtesy Postdoctoral Associate, Florida International University, Miami, FL, USA \\ Email: JLparra@fiu.edu
}

How to cite this paper: Parra, J.L. (2018) Corpuscular Point of View to Explain Light's Properties. Optics and Photonics Journal, 8, 135-145.

https://doi.org/10.4236/opj.2018.85013

Received: March 23, 2018

Accepted: May 28, 2018

Published: May 31, 2018

Copyright $\odot 2018$ by author and Scientific Research Publishing Inc. This work is licensed under the Creative Commons Attribution International License (CC BY 4.0).

http://creativecommons.org/licenses/by/4.0/

\section{(c) (i) Open Access}

\begin{abstract}
This letter introduces a simple model to explain the Diffraction and Interference of Light. It was created using only a corpuscular point of view. The mean concept of the model introduced in this paper is that light has two independent states of polarization that oscillate with equal frequencies but with a $\pi / 2$ difference of phase. This model allows the author to determine the intensity of light at any point after it exceeds no edge or any number of them.
\end{abstract}

\section{Keywords}

Diffraction, Interference, Photons, Mach-Zehnder Interferometer

\section{Introduction}

Currently, all properties of light cannot be explained using a sole and simple point of view. On one hand, Newton's image [1] that light is made of particles is required to explain the photoelectrical effect [2] and Compton's effect [3]. On the other hand, Huygens's waves [4] accommodate very well to explain patterns of diffraction and interference [5]. Both paradigms of light stem from two irreconcilable points of view that create an uncomfortable situation. For that reason, the accepted criterion has been that light is a combination of waves and particle properties, it is something different from everything known in the macroscopic world.

In this paper, the sequence of maxima and minima of light will be explained by using a pure and corpuscular point of view. A revision of the accepted properties of light can help to approach this plan. In 1901, Max Planck [6] initiated a new era in physics by finding the density of the number of states available to an ensemble of oscillators (see Equation 12 on [6]); there is a coefficient of value 8 . Bose [7] obtained the same distribution following another statistical approach but finished with a coefficient of 4 under the assumption that every photon can 
only have one state of polarization. Einstein proposed to solve the problem by assuming that the photon electric field has two independent components that duplicate the number of cells that can be present in every statistical state. Einstein's idea is hard to visualize and even Bose shows some skepticism by expressing "To take into account polarization it appears necessary to multiply this number by 2" [7]. New experimental outcomes move Hutchin [8] to conclude that the vector potential is real and should be understood as another form of polarization of light, it is a new third state. According to Hutchin [8], this third polarization and the electrical field polarization should interact with matter in a different and independent, way. If that is correct, then the coefficient in Planck's equation should be 12, which would be incompatible with the original value of 8 . The original thoughts from Planck, Bose, Einstein, and Hutchin cannot be totally harmonized. However, the four points of view can be reconciled with a little variation, which could be achieved by assuming that the two-polarizing directions, claimed by Einstein, are the electrical field and the vector potential fields. In that way the four authors pointed out coefficients with the same value of 8 . This paper is, then, the study of the consequences of these two polarizations assuming that both have a wavelength of $\lambda$ but separate in phase by $\pi / 2$ (Hypothesis One).

Hypothesis Two: Two photons that are close to each other with transversal and longitudinal polarizations in phase will experience a plastic attraction; while two photons with corresponding polarizations in opposition of phase will experience an elastic repulsion.

These two hypotheses explain why the spatial coherence of light increases in distance after photons re-accommodated inside a pack according to their relative phases. While the photons travel, if any perturbation occurs the phase of one or more photons, they will slowly move out of the group and the pack's direction would continue despite the disruption. The outside photons shield the others so that the pack can travel in harmony as a peloton. This idea can help us to understand why pictures of galaxies are clear.

This model will be used to explain diffraction. Suppose some photons are moving down vertically and perpendicularly to a horizontal slit the size of a. Then, photons that are moving close by the edge of the slit will experience one of two possibilities: 1) a sort of refraction and a turning away from the symmetry axis of the slit; or 2) a sort of reflection and bounce in the direction of the axis of the slit. All photons that suffer reflection will experience a phase increase of pi. The interaction between photons that suffered reflection with photons that move in a straight line will create the Fresnel's diffraction zone [9]. Outside of the central part of the slit and far away from it, photons that suffered refraction will meet with photons that suffered reflection, and they will create what is known as Fraunhofer's diffraction [9].

\section{Intensity}

The electrical field intensity in a region that is located outside of a flat surface is 
inversely related to the distance $r$. The theoretical calculation of the cross section for particles approaching that kind of field is extremely difficult. In this paper, an approximate calculation was used to save time. It consists of a program with infinitesimal displacements that mimic photon trajectories after they interact with the electrical field. In this calculation, it was assumed that some of photons are attracted by the electrical field and others are repelled. The quantity of photons attracted and repelled will be explained later in this paper. The author's program works with enough statistical interactions to get a clear function distribution of particles on bins of one degree. The program was run to a reasonable distance from the edge for the necessary amount of time to accumulate enough information. This is more effective because going to infinity will naturally accumulate infinity particles with deviation between zero and one degree. As it is known, that intensity $I$ is related to the density of particles; the following function describes the calculated angle distribution of particles in both directions, at any point far away after the perturbation $I=\frac{I_{o}}{\sin ^{2}\left(\frac{\theta}{2}\right)}$.

Moreover, any interaction between coming particles with any weak field will show some measurable effect only after the particles travel a long distance. In this model, a cut off distance definition-Y Initial $\left(Y_{I n i}\right)$-was used to avoid the infinite effect mentioned before. Consequently, if a screen is placed at some distance $x$ from the field's plane, any particles going through the field at a distance $y$ bigger than $Y_{I n i}$ will collide with that screen quasi-perpendicularly. At this point the $Y_{I n i}$ value will depend on the distance $x$. Any point of the screen with $y$ bigger than $Y_{I n i}$ can have photons simultaneously coming straight and photons affected by the repulsion of the edge. The closer the photons move around the edge, the stronger the repulsion they will experience and the longer the distance they will travel to get to the screen. The final distance travelled by the photon after repulsion $\left(Y_{f r}\right)$ follow the equation $y_{f r}=y-y_{I n i} \mathrm{e}^{\frac{y_{l i n}-y}{x}}$ (extreme values for this equation are $\mathrm{y}_{f r}=0$ for $y=y_{I n \dot{p}}$ and $y_{f r}=y$ for $\left.y-y_{I n i} \ll x\right)$. However, when calculating the photon's angle of deviation $(\theta)$, the vision line makes us "believe" the photon comes from the edge; thus, the equation that might be used to calculate the angle is $\tan \theta=\frac{y}{x}$. These last two equations are key points for understanding light behavior.

\section{One Edge}

Let's assume that all photons arriving simultaneously to the flat field plane have same null phase to simplify the math. It is, photons moving within quasi-parallel rays and instantaneous occupying the same plane, will have same phase, in this case null phase. No mathematical elaboration will be used, to focus on the model presented here. The calculations will be divided in two regions because the physics of the problem follow two different combinations. The shadowed region (1) 
where $y$ is lower than $Y_{I n p}$ and the illuminated region (2) where $y$ is bigger than $Y_{\text {Ini }}$

\subsection{Illuminated Region}

The intensity can be calculated using the superposition of the electrical field in the transversal direction, and the superposition of the vector potential in the longitudinal direction. Photons moving straight will merge with photons effected by repulsion on the edge. The simplified expression of the intensity, without intentional trigonometrical reduction, becomes:

$$
I_{I}=I_{0}\left\{\left[\cos \left(\varphi_{o}\right)+\frac{A_{I} \cos \left(\varphi_{1}\right) \sin \left(\theta_{0}\right)}{\sin \left(\theta_{I}\right)}\right]^{2}+\left[\sin \left(\varphi_{o}\right)+\frac{A_{I} \sin \left(\varphi_{1}\right) \sin \left(\theta_{0}\right)}{\sin \left(\theta_{I}\right)}\right]^{2}\right\}
$$

where $\varphi_{o}=\frac{2 \pi}{\lambda} x, \quad w=\frac{0.8 x}{a \sqrt{\frac{2 \pi}{\lambda}\left(\frac{1}{a}+\frac{1}{b}\right)}}, \quad \varphi_{1}=\varphi_{I n i}+\frac{2 \pi}{\lambda} \sqrt{x^{2}+(w v)^{2}}$,

$\theta_{o}=\tan ^{-1}\left(\frac{y_{I n i}}{x}\right), \theta_{I}=\tan ^{-1}\left(\frac{w v}{x}\right), A_{I}$ is the amplitude "reflected," $\lambda$ is the "spatial period"-distance between closer photons on a beam with same phase and equivalent to the wavelength on the wave theory-and $v$ is a unitless parameter proportional to the distance from the geometric shadow to the point where the intensity has been calculated. $v$ is well documented in [9] [10] [11] and it is fraction of some geometrical parameters, that also include the spatial periodicity $\lambda$.

\subsection{Shadowed Region}

The intensity of the shadowed region obeys:

$I_{S}=I_{0}\left\{\left[\frac{A_{S} \cos \left(\varphi_{2}\right) \sin \left(\theta_{0}\right)}{\sin \left(\theta_{S}\right)}\right]^{2}+\left[\frac{A_{S} \sin \left(\varphi_{2}\right) \sin \left(\theta_{0}\right)}{\sin \left(\theta_{S}\right)}\right]^{2}\right\}=I_{0}\left\{\left[\frac{A_{S} \sin \left(\theta_{0}\right)}{\sin \left(\theta_{S}\right)}\right]^{2}\right\}$

where $\theta_{S}=\tan ^{-1}\left(\frac{w v-v_{I n i}}{x}\right)$. Equations (1) and (2) include the parameter $Y$ initial that could be considered as a constant of integration. It will guarantee the continuity between the shadow and illuminated functions. Figure 1 shows these requirements. This graph can be compared to the one on page 57 of [9], considering that their graph represents the amplitude of the electrical field and not the intensity. This calculation describes what is known as Fresnel's Diffraction. J.D. Barnett and F.S. Harris, Jr., utilized Equation (2) [10] to calculate Fresnel's Diffraction, which I will use in this work as well. Figure 1 includes a superposition of that equation, some experimental points from [11], and the author's illuminated and shadow regions using the parameters $I_{0}=1$, $\lambda=546.1 \mathrm{~nm}, a=20.243 \mathrm{~m}, b=32.632 \mathrm{~m}, x=a+b, V_{I n i}=5, \varphi_{I n i}=-2.7 \mathrm{rad}, A_{I}=$ 0.16 , and $A_{S}=0.5$. 


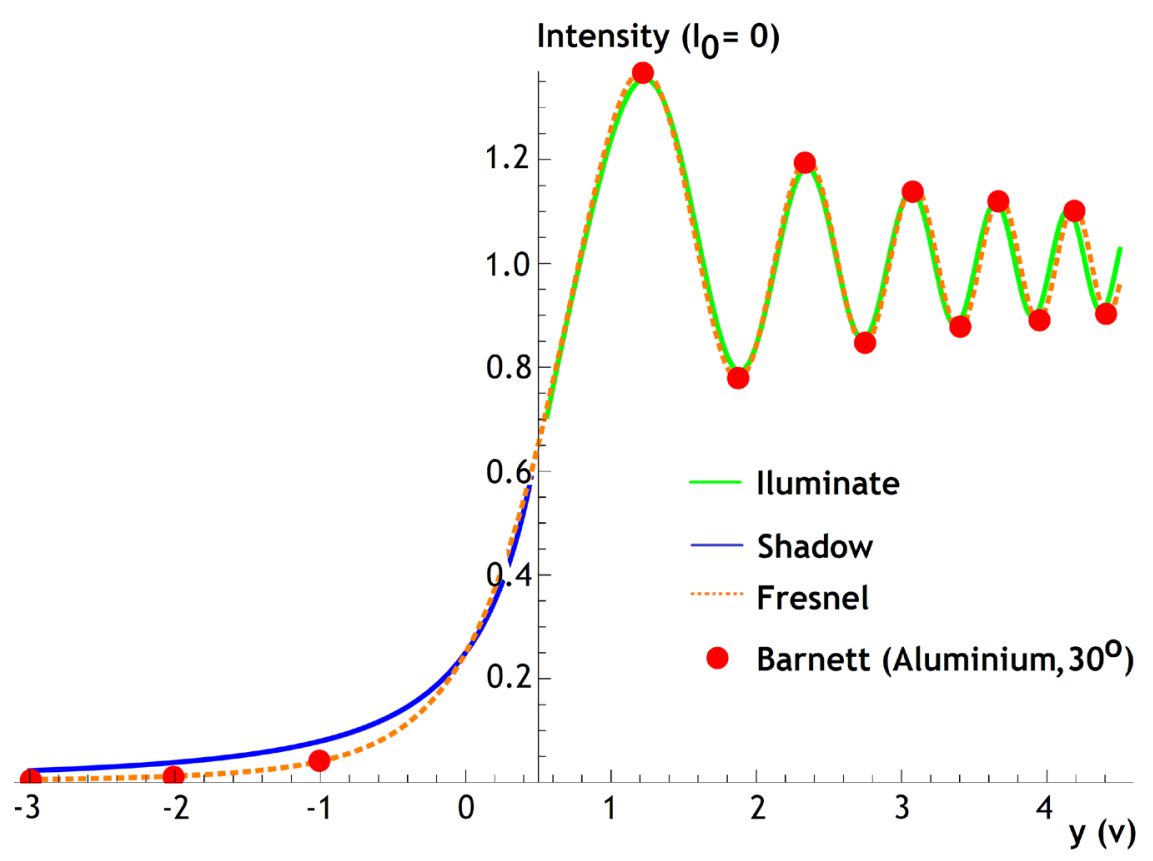

Figure 1. Open intensity normalize to one. The Barnett's experimental points can be founded in his first columns of Tables VI and VII [11].

\section{Two Edges}

There is a symmetry line between the two edges that will be used as a frame of reference. Both edges define a slit of width $a$ and, at a perpendicular distance far away from it, the intensity pattern is described by Fraunhofer's equation. On a screen located at a distance $x$ from the slit with the condition $x \gg a$, all photons coming through it will NOT arrive perpendicular to it because of the interaction with the edges. This situation slightly changes the physics of this process thus, it is important to assess these changes. The value of $Y$ initial increases while the base angle $\theta_{0}$ decreases on Fresnel's region with the increment of the distance $x$. Fraunhofer's region starts when $Y$ initial stops growing at the possible maximum value (half of the slit width) and the base angle stops decreasing at the minimum value. In Fresnel's region, photons are moving simultaneously in $x$ and $y$ directions producing a cylindrical symmetry, while in Fraunhfer's region these photons are somehow moving in $z$ direction producing a spherical symmetry. Intensity on the last case is also modulated by a fraction. The numerator of the intensity exhibits a "dispersive spherical" effect: $\frac{\lambda^{2}}{4 \pi^{2}\left(\frac{a}{2}\right)^{2}}$ The denominator of the intensity shows a sinusoidal form, which raised to the second power will look like this $\left(\frac{2 y}{\sqrt{x^{2}+y^{2}}}\right)^{2}$. Normalizing the ratio of both parts can be achieved by introducing a constant in the modular function $f_{N}$. Then, according to the identity $\sin (\varphi)=\cos (\varphi+\pi / 2)$, the intensity could be described by Equation (3), 


$$
I=f_{N}\left\{\left[\cos \left(\varphi_{1}\right)+\cos \left(\varphi_{2}\right)\right]^{2}+\left[\sin \left(\varphi_{1}\right)+\sin \left(\varphi_{2}\right)\right]^{2}\right\}
$$

where $\varphi_{1} \approx \pi+\frac{2 \pi}{\lambda} \sqrt{x^{2}+\left(\frac{a}{2}+y\right)^{2}}$ for "reflected" photons from the left edge (exact value of the phase will not change the final numerical result) and $\varphi_{2} \approx \frac{2 \pi}{\lambda} \sqrt{x^{2}+\left(\frac{a}{2}-y\right)^{2}}$ for "refracted" photons from the right edge.

The normalized function results in $f_{N}=\left(\frac{\lambda x}{2 \pi a y}\right)^{2}$ after applying approximate calculations when $y$ comes closer to zero. Figure 2 shows a graphical comparison between the intensities calculated using the equation introduced in this paper and the following recognized function: $I=I_{o}\left\{\frac{\sin \left[\frac{\pi a}{\lambda} \sin (\theta)\right]}{\frac{\pi a}{\lambda} \sin (\theta)}\right\}^{2}$

\section{Many Edges}

At this point, the physics of this paper have been all set. However, it is important to analyze what happens when there are many slits involved. Now, the more convenient reference frame should be centered according to the symmetry defined by the total number of edges. On 5 slits, photons coming from reflection and refraction contact each other with the phases:

$\varphi_{n}^{1} \approx \pi+\frac{2 \pi}{\lambda} \sqrt{x^{2}+\left[y+n d+\frac{a}{2}\right]^{2}}$ and $\varphi_{n}^{2} \approx \frac{2 \pi}{\lambda} \sqrt{x^{2}+\left[y+n d-\frac{a}{2}\right]^{2}}$ where $n$ goes from -2 to 2 ; there are five additions of each kind. The $d$ is the distance between the centers of two adjacent slits. Intensity represented in Figure 3 comes from

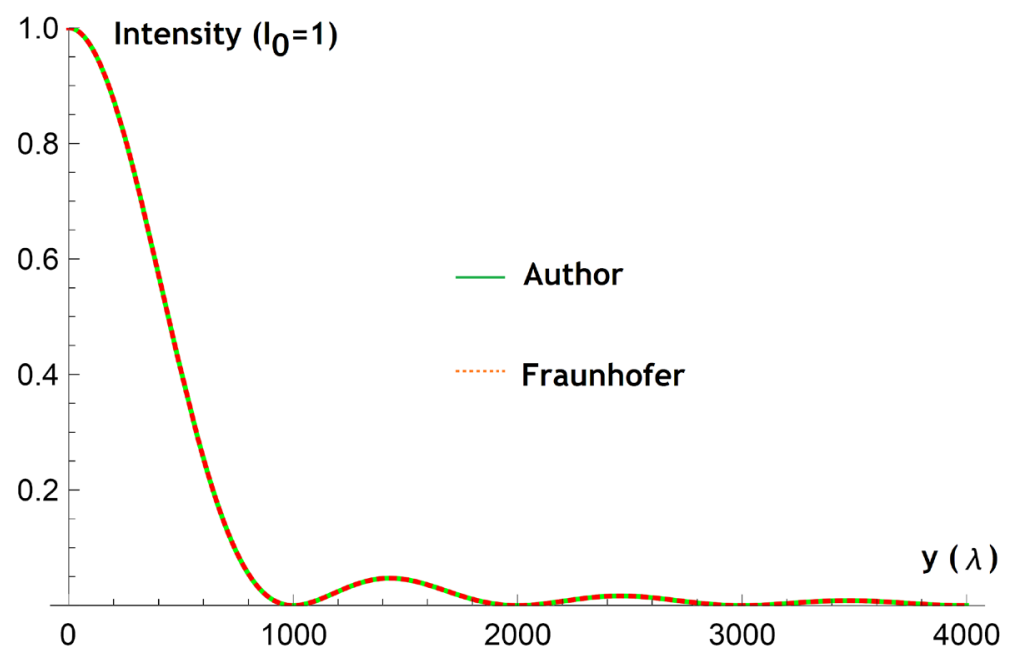

Figure 2. Both functions with maxima intensity were normalized to one. The intensity was calculated at distance $x$ equal to a million spatial periods behind the slit. The slit width was equal to a thousand spatial periods of value one. 


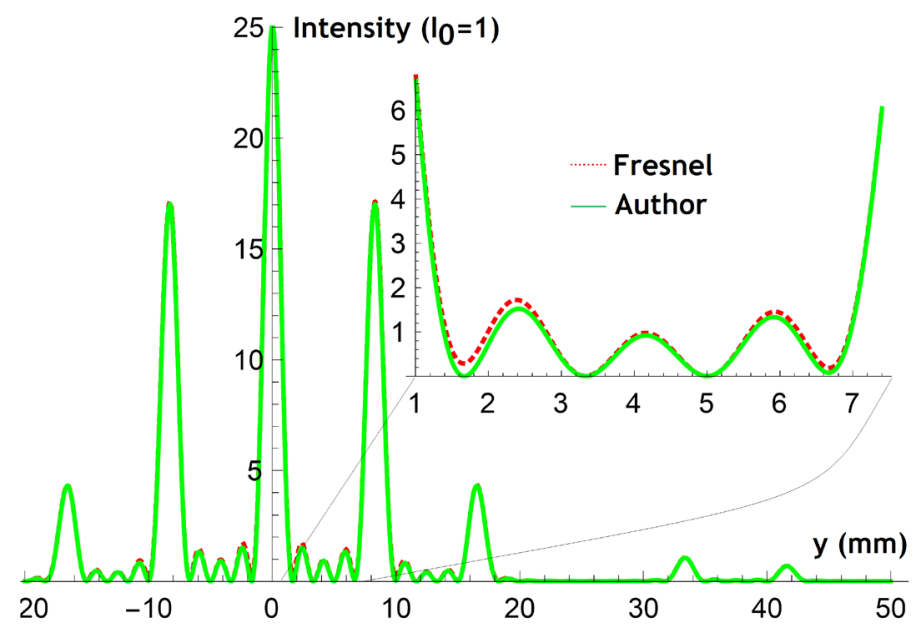

Figure 3. Photons coming through five slits. The precise calculation of the actual wave theory was conducted using Fresnel's equations from the program Wolfram Mathematica 10. See function on the Appendix.

Equation (4), and the parameters $\lambda=0.0005 \mathrm{~mm}, x=2000 \mathrm{~mm}, a=0.04 \mathrm{~mm}$, and $d=0.12 \mathrm{~mm}$.

$$
I=\left(\frac{x \lambda}{2 \pi a y}\right)^{2}\left(\left\{\sum_{n=-2}^{n=2}\left[\cos \left(\varphi_{n}^{1}\right)+\cos \left(\varphi_{n}^{2}\right)\right]\right\}^{2}+\left\{\sum_{n=-2}^{n=2}\left[\sin \left(\varphi_{n}^{1}\right)+\sin \left(\varphi_{n}^{2}\right)\right]\right\}^{2}\right)
$$

Figure 3 shows the four basic properties described in the book [12]. The first property is $I_{\max }=N^{2} I_{\mathrm{o}}$ (where $N$ is the number of slits and $I_{o}$ is the maximum intensity for one slit). The second one is that the number of secondary maxima equals $N-2$. The third one expresses the principal maxima are exhibited on points which obeys equation $d \sin (\theta)=m \lambda$. The fourth one is that the minima occurs at points which obeys equation $\operatorname{asin}(\theta)=m \lambda$.

The increment of the number of slits increases height and thickness of the principal maxima. The more edges you have, the greater the number of photons that will go through them and the bigger the chance to force each other to go in a different direction until they found a direction where photons around them have the same phase. On gratings, the number of slits is so big that the huge number of minima removes most of the photons between maxima.

\section{Mach-Zehnder Interferometer}

On this device, the diffraction is eliminated allowing the study of pure interference. This produces higher intensities and a clearer manifestation of the interactions between photons. The apparatus by itself is more sophisticated than a superficial option such as a screen with two holes; however, the mathematical output is dramatically simplified because of the necessity to pay attention to only two virtual foci. The two outputs in the Mach-Zehnder interferometers come from the same unique source. But, if anybody remove one of the sensors, and look in the direction of the coming light, it will give the impression that the two beams come from two apparent sources, because both beams come from differ- 
ent directions. Mathematically, it is possible to replace the bouncing real paths with two imaginary straight lines of the same optical length. Equation (5) included the interaction between the two virtual straight beams; and Equation (6) is almost the same but adds a $\pi$ increment of phase to one of the beams as is proposed in [13],

$$
\begin{gathered}
I=I_{0}\left\{\left[\cos \left(\varphi_{1}\right)+\cos \left(\varphi_{2}\right)\right]^{2}+\left[\sin \left(\varphi_{1}\right)+\sin \left(\varphi_{2}\right)\right]^{2}\right\} \\
I=I_{0}\left\{\left[\cos \left(\pi+\varphi_{1}\right)+\cos \left(\varphi_{2}\right)\right]^{2}+\left[\sin \left(\pi+\varphi_{1}\right)+\sin \left(\varphi_{2}\right)\right]^{2}\right\}
\end{gathered}
$$

where $\varphi_{1} \approx \frac{2 \pi}{\lambda} \sqrt{x^{2}+(y+b)^{2}+(z+c)^{2}}$ and $\varphi_{2} \approx \frac{2 \pi}{\lambda} \sqrt{x^{2}+y^{2}+y^{2}}$. Figure 4 shows three different combinations of the offset $b$ and $c$. On left set $b \neq 0$ and $c=$ 0 ; on middle set $b=0$ and $c \neq 0$, and on the right set $b \neq 0$ and $c \neq 0$.

Particularly interesting to study is what happened to the Mach-Zehnder outputs if the difference of path between the beams becomes closer to zero. How the output will change if a miniscule perturbation occurs in some place of the body of any of the beam splitters? If the perturbation occurs in the first beam splitter both outputs should reflect the perturbation, as is visible on the right-top corner of Figure 5. This situation induces the incorrect interpretation that the photons

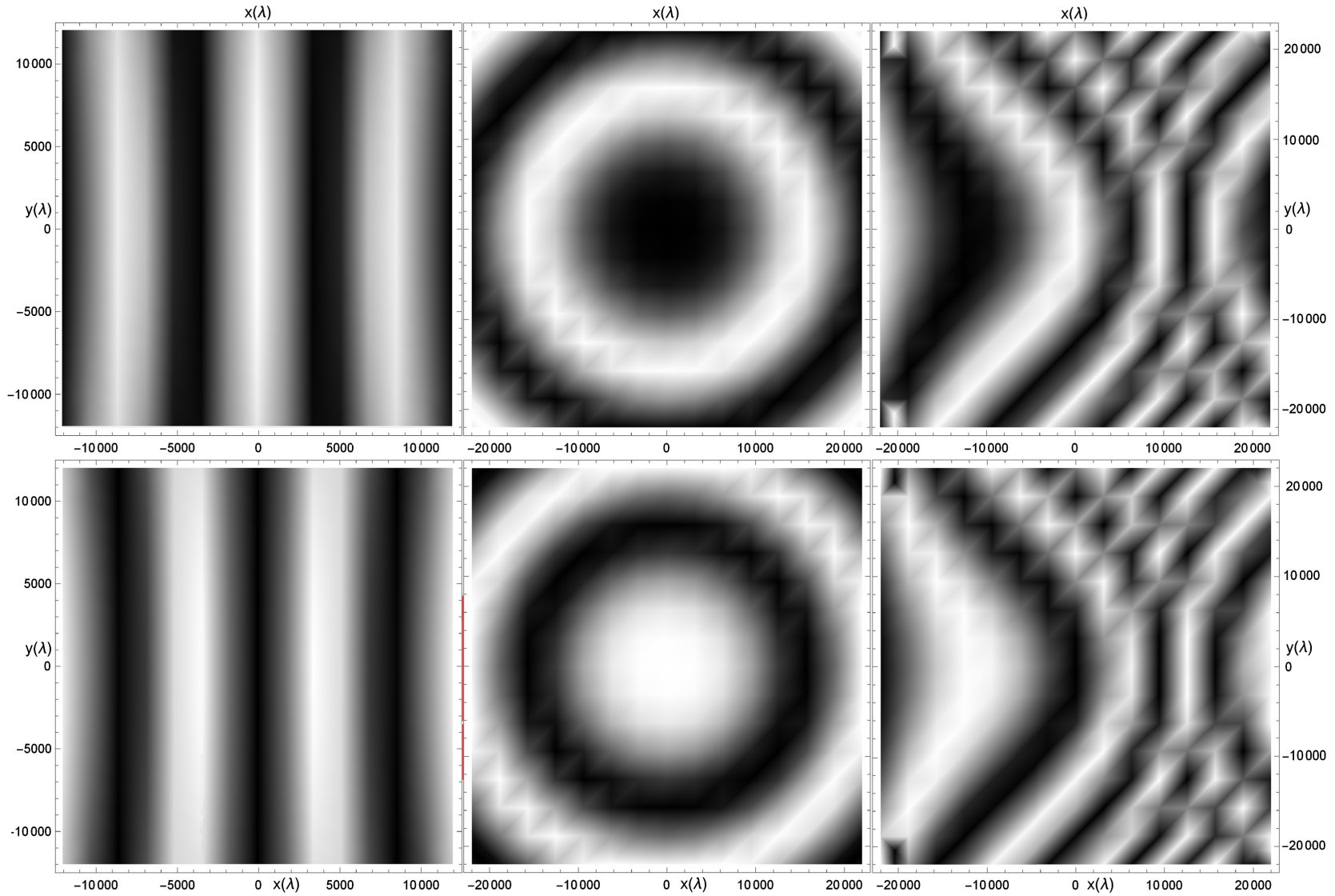

Figure 4. Mach-Zehnder outputs with the two virtual foci in three positions: 1) both foci perpendicular to the line of observation; 2) both foci in aliment with the line of observation; and 3) a combination of the previous ones. 


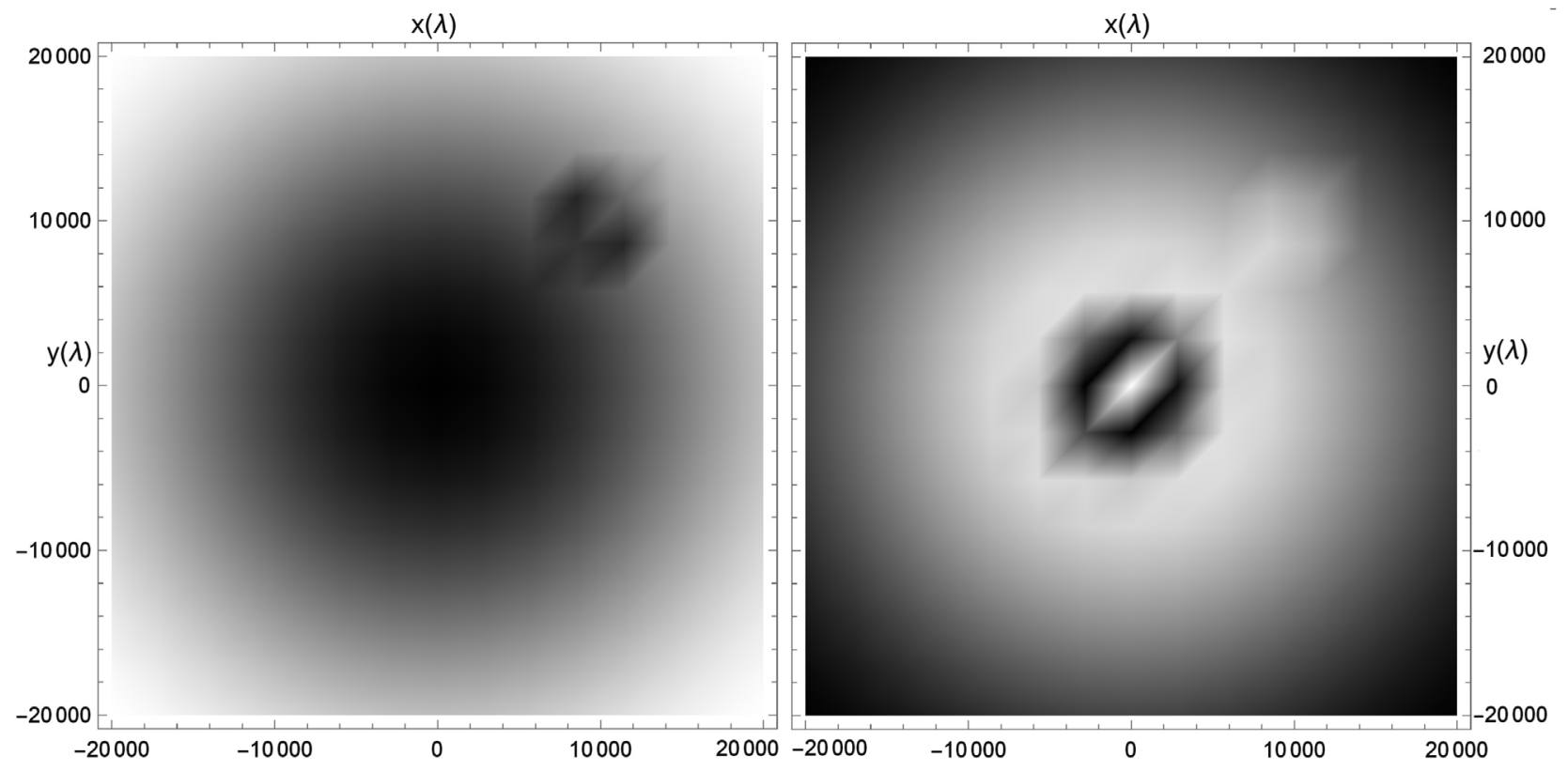

Figure 5. Mach-Zehnder outputs from two virtual foci in aliment with the line of observation and with almost zero difference of path between both beams. The consequence of the perturbation on the first beam splitter is visible on the upper right corner of each of the images. The consequence of perturbation on the second beam splitter is visible in the center of the right output image but none is seen in the left one. Experimental comparison could be done with the Figure 7 and Figure 8 of the author's previous paper [14].

that are missing in one output come to the other one. The correct interpretation should be that the photons that are pushed away from a repulsive interaction (destructive interference on the wave theory) come to the surrounding space. This case can be observed in the center of the figure of the right output where the black spot does not match a brilliant one in the left output because the perturbation occurs in the second beam splitter.

\section{Conclusions}

Currently, there are contradictions between different theories within the microscopic world, which have decreased the intrinsic harmony of Physics. To minimize these theoretical contradictions, physicists created an artificial model that has made satisfied some and made skeptics of others. Some of these contradictions come into question while one attempts to explain the nature of light. It is viewed as a mixture of wave-particles properties. In this paper, the vast world of diffraction and interference of light was explained with a simple idea based on photon interactions. These interactions are modulated by assuming that photons have two orthogonal polarizations: one transversal to and the other longitudinal to the line of propagation. Three main interactions produce all the variation of the light's intensity: pure attraction, a combination of attraction and repulsion, and pure repulsion. The first one defines the maxima while the last one defines the minima on any intensity distribution. From a practical point of view, where the condition of minimum is satisfied, photons are pushed away from one 
another. However, where the condition of maximum is satisfied, the coherence between photons increases; photons can travel staying as close as possible to each other and forcing each other to be in phase. In some way, a group of photons is comparable to a perfect elastic body, until some strong enough interactions appear and separate one or more of them from the group. The author model uses two orthogonal directions as are done on the accepted wave theory of light. But these two points of view are not equivalents to each other at all, no mathematically not physically, because one requires the integrals of many secondary emissions, and the author one only asks for the addition of many sources as edges are involved on the problem.

In short, there is no need of using a wave point of view or any wave's properties to explain the diffraction or interference of light. Since Calculus was not used, the content of all these ideas could be explained, if it is desired, to even beginning students of physics at the middle school level. If this is true, maybe it will become necessary to re-interpret de Broglie's ideas [15].

\section{Acknowledgements}

My sincerest gratitude goes to Thelma Cosme and Natalie Garcia. Their efforts aided the careful editing and correction in the style of delivery for every idea introduced throughout the body of this paper.

\section{References}

[1] Newton, I. (1704) Opticks. London.

[2] Einstein, A. (1905) On a Heuristic Viewpoint Concerning the Production and Transformation of Light. Annalen der Physik, 17, 132-148. https://doi.org/10.1002/andp.19053220607

[3] Compton, A.H. (1923) A Quantum Theory of the Scattering of X-Rays by Light Elements. Physical Review, 21, 483-502. https://doi.org/10.1103/PhysRev.21.483

[4] Huygens, C. (1690) Tratě de la Lumiere. Pierre Vander Aa, Ghent University.

[5] Young, Th. (1802) Philosophical Transactions of the Royal Society. Young's Works, London xcii, 12,387.

[6] Planck, M. (1901) On the Law of Distribution of Energy in the Normal Spectrum. Annalen der Physics, 4, 553. https://doi.org/10.1002/andp.19013090310

[7] Bose, S.N. (1924) Planck's Law and Light Quantum Hypothesis. Zeits. Physik, 26, 178. https://doi.org/10.1007/BF01327326

[8] Hutchin, R.A. (2015) The Third Polarization of Light. Optics and Photonics Journal, 5, 29-32. https://doi.org/10.4236/opj.2015.52004

[9] Born, M. and Wolf, E. (1980) Principles of Optics. 6th Edition, Pergamon Press, New York.

[10] Barnett, J.D. and Harris, F.S.Jr. (1961) Test of the Effect of Edge Parameters on Small-Angle Fresnel Diffraction of Light at a Straight Edge. JOSA, V52-N6.

[11] Barnet, J.D. (1959) Effect of Edge Parameters on Fresnel Diffraction of Light at a Straight Edge. Ph.D. Thesis, University of Utah, Salt Lake City.

[12] Young, H.D. and Freedman, R.A. (2004) Sears and Zemansky's University Physics 
with Modern Physics. 11th Edition, Pearson Addison Wesley, San Francisco, 1374.

[13] Zetie, K.P., Adams, S.F. and Tocknell, R.M. (2000) How Does a Mach-Zehnder Interferometer Work? Physics Education, 35, 46.

https://doi.org/10.1088/0031-9120/35/1/308

[14] Parra, J.L. (2018) Single-Photon Interaction with Beam Splitters. Optics and Photonics Journal, 8, 20-28. https://doi.org/10.4236/opj.2018.82003

[15] de Broglie, L. (1924) Researches on the Quantum Theory. Ph.D. Thesis, Paris.

\section{Appendix}

Plot[160((-FresnelS[z-.52]+FresnelS[z-.44]-FresnelS[z-.28]+FresnelS[z-.2]-Fresnel $\mathrm{S}[\mathrm{z}-.04]+$ FresnelS$[\mathrm{z}+.04]$-FresnelS $[\mathrm{z}+.2]+$ FresnelS $[\mathrm{z}+.28]$-FresnelS $[\mathrm{z}+.44]+$ Fresne $1 \mathrm{~S}[\mathrm{z}+.52])^{\wedge} 2$

(-FresnelC[z-.52]+FresnelC[z-.44]-FresnelC[z-.28]+FresnelC[z-.2]-FresnelC[z-.04] + FresnelC $\left[\mathrm{z}^{+} .04\right]$-FresnelC $[\mathrm{z}+.2]+$ FresnelC $[\mathrm{z}+.28]$-FresnelC $[\mathrm{z}+.44]+$ FresnelC $[\mathrm{z}+$. $\left.52])^{\wedge} 2\right),\{z,-20,50\}$, PlotRange- $>\{0,25\}$,

PlotStyle- $>\{$ Red,Dashing[Tiny] $\}$, PlotLegends- $>\{$ "Fresnel" $\}]$; 